\title{
$\$$ Research Square \\ Regulation of Adropin by Sitagliptin Monotherapy in Participants With Newly Diagnosed Type 2 Diabetes
}

Qiu Wang

Beijing Chao-Yang Hospital

Yu An

Beijing Chao-Yang Hospital

Lin Zhang

Beijing Chao-Yang Hospital

Yuanying Zhang

Beijing Chao-Yang Hospital

Guang Wang

Beijing Chao-Yang Hospital

Jia Liu (D liujia0116@126.com )

Beijing Chao-Yang Hospital

\section{Research Article}

Keywords: adropin, sitagliptin, type 2 diabetes

Posted Date: March 8th, 2022

DOI: https://doi.org/10.21203/rs.3.rs-1275332/v1

License: (c) (1) This work is licensed under a Creative Commons Attribution 4.0 International License.

Read Full License 


\section{Abstract}

Background: Adropin is a potent metabolic regulator of insulin sensitivity and glycolipid metabolism. The present study investigated the effects of sitagliptin on adropin and metabolic parameters in participants with newly diagnosed type 2 diabetes (T2D).

Methods: Thirty-five participants were diagnosed with T2D and were prescribed sitagliptin $100 \mathrm{mg}$ once daily for 17 weeks. Twenty-eight age-, sex-, and BMI-matched healthy subjects were included as the control group. Adropin and clinical parameters were assessed at baseline and after treatment.

Results: Serum adropin levels were lower in T2D participants than in the healthy individuals $(3.12 \pm 0.73$ vs. $5.90 \pm 1.22 \mathrm{ng} / \mathrm{ml}, P<0.01)$. Serum adropin levels were significantly increased in T2D patient after sitagliptin treatment $(4.97 \pm 1.01$ vs. $3.12 \pm 0.73 \mathrm{ng} / \mathrm{ml}, P<0.01)$. The changes in serum adropin levels after sitagliptin treatment were parallel with the improving of fasting blood glucose (FBG) $(\beta=-0.71, P<$ $0.01)$, glycosylated hemoglobin (HbA1c) $(\beta=-0.44, P<0.01)$ and homeostatic model assessment of $\beta$ cell function (HOMA- $\beta)(\beta=9.02, P<0.01)$.

Conclusions: Sitagliptin treatment significantly increased serum adropin levels in participants with newly diagnosed T2D. And the changes in serum adropin levels were parallel with the amelioration of glucose metabolism.

Trial registration: Clinicaltrials.gov, NCT04495881. Retrospectively registered on 3 August 2020.

\section{Introduction}

Adropin is a unique peptide hormone firstly reported in 2008. It contains 76 amino acids encoded by Energy Homeostasis Associated (Enho) gene and is predominantly expressed in liver and brain ${ }^{1}$. Some animal studies have shown that adropin is involved in the control of glycolipid metabolism and the improvement of insulin sensitivity ${ }^{1-4}$. Lower serum adropin levels in human have been proved to be associated with multiple metabolic disorders such as type 2 diabetes (T2D), nonalcoholic fatty liver disease (NAFLD), polycystic ovary syndrome (PCOS) and metabolic syndrome $e^{5-8}$.

T2D is a chronic metabolic disorder characterized by chronic hyperglycemia due to $\beta$-cell dysfunction and insulin resistance ${ }^{9}$. Sitagliptin is approved for T2D therapy and acts by inhibition of dipeptidyl peptidase4 (DPP-4) enzyme ${ }^{10}$. DPP-4 is expressed in different tissues such as liver, brain, and adipose tissue ${ }^{11-13}$. However, to our knowledge, how sitagliptin regulates serum adropin is unknown. Therefore, we investigated the changes in serum adropin levels in participants with newly diagnosed T2D following sitagliptin treatment.

\section{Materials And Methods \\ 2.1 Study design}


This was a phase 4, open-label, single-arm, interventional study to evaluate the effectiveness of sitagliptin for the treatment of newly diagnosed T2D. The study was conducted in accordance with the Declaration of Helsinki and was registered on Clinicaltrials.gov on 3 August 2020 (registration number NCT04495881). The protocol was approved by the Ethics Committee of Beijing Chao-yang Hospital affiliated with Capital Medical University (2020-\#-182). All enrolled participants provided written informed consent.

\subsection{Recruitment}

Thirty-eight participants with newly diagnosed T2D (T2D group) were recruited from outpatients at the Endocrinology Department of Beijing Chao-yang Hospital affiliated with Capital Medical University from January 2020 to March 2020. They met inclusion and exclusion criteria, which were similar to those used in our previous studies ${ }^{14}$. All participants underwent medical screening including a $75 \mathrm{~g}$ oral glucose tolerance test (OGTT). Eligible participants aged 18 to 65 years were newly diagnosed with T2D within the previous 3 months according to the 2014 American Diabetes Association (ADA) diagnostic criteria and they were further selected when $\mathrm{HbA} 1 \mathrm{c} \geq 7.0 \%$ and $\leq 9.5 \%{ }^{15}$. They didn't use any hypoglycemic drugs before the enrolment. The healthy control group included twenty-eight age-, sex-, and BMI-matched healthy participants with normal glucose tolerance who received routine physical examination. We excluded all participants whose $\mathrm{HbA1c}$ levels felt out of our selection ranges and those who had type 1 diabetes mellitus, pancreatitis, pregnancy or possible pregnancy, liver or renal function impairment, coronary heart disease, infectious disease, history of intestinal surgery, chronic hypoxic diseases, hematological disease, systemic inflammatory disease and cancer.

The T2D participants orally received sitagliptin $100 \mathrm{mg} /$ day for 17 weeks. Furthermore, all participants were given advice for lifestyle modifications in diet and physical activity. Drug compliance, vital signs and adverse events were monitored by outpatient review or telephone follow-up. The patients who have poor glycemic control or could not accept the follow-up visit were withdrawn from the study.

\subsection{Clinical and biochemical measurements}

The participants with T2D were followed-up for 17 weeks. The fasting blood samples were taken before and after the 17-week sitagliptin treatment. The serum samples from all participants were collected in the morning after an overnight fast and stored at $-80^{\circ} \mathrm{C}$ until analysis.

Anthropometric measurements and biochemical laboratory tests were performed at baseline (pretreatment) and after 17 weeks of sitagliptin treatment (post-treatment). Weight and height were measured to the nearest $0.1 \mathrm{~kg}$ and $0.1 \mathrm{~cm}$ in the fasting state, respectively. FBG, HbA1c, fasting insulin (FINS), total cholesterol (TC), low-density lipoprotein cholesterol (LDL-C), high-density lipoprotein cholesterol (HDL-C), and triglycerides (TG) were measured at the central chemistry laboratory of Beijing Chao-yang Hospital affiliated with Capital Medical University. The primary outcome measure was the changes in $\mathrm{HbA} 1 \mathrm{c}$ at baseline and after 17 weeks treatment, and the secondary outcome measures included change in FBG, FINS, HOMA-IR, HOMA- $\beta$, TC, HDL-C, TG and LDL-C. 
Serum adropin levels were measured using commercial enzyme-linked immunosorbent assay (ELISA) kits (EK-032-35, Phoenix Pharmaceuticals, Inc., USA) for quantitative detection. The sensitivity of the assay was $0.32 \mathrm{ng} / \mathrm{ml}$ and the linear range of the standard was $0.32-5.80 \mathrm{ng} / \mathrm{ml}$. BMI was calculated as the weight in kilograms divided by the height in meters squared $\left(\mathrm{kg} / \mathrm{m}^{2}\right)$. Homeostatic model assessment of insulin resistance (HOMA-IR) and HOMA- $\beta$ were calculated by the following formulas: $\mathrm{HOMA-IR}=\mathrm{FINS}$ $(\mu \mathrm{IU} / \mathrm{mL}) \times \mathrm{FBG}(\mathrm{mmol} / \mathrm{L}) / 22.5 ; \mathrm{HOMA}-\beta=20 \times \mathrm{FINS}(\mu \mathrm{IU} / \mathrm{mL}) / \mathrm{FBG}(\mathrm{mmol} / \mathrm{L})-3.5^{16,17}$.

\subsection{Statistical analysis}

The data were performed using the SPSS statistical software, version 23.0 (SPSS Inc., Chicago, IL, USA). The normality of distribution was verified by Shapiro-Wilk test. The data were expressed as means \pm standard deviation (SD) or median (interquartile range). The Student's $t$-test or nonparametric MannWhitney U-test for continuous variables was used to compare the differences between healthy controls and T2D participants. Statistical differences between the untreated T2D participants and after a 17-week of pharmacotherapy were verified by the paired Student's $t$-test or nonparametric Wilcoxon test. Pearson and Spearman correlation coefficients were used to assess the correlations between serum adropin levels and other metabolic parameters at baseline as appropriate. We fit linear mixed-effects models using the STATA version 13.0 (STATA, College Station, TX), allowing for the inclusion of individual as a random effect, to examine the longitudinal relationship between serum adropin levels and insulin resistance/sensitivity indicators as well as parameters of glucose-lipid metabolism over the study period. Mixed effects linear models can account for the associations between repeated measures owing to unobserved inter-individual heterogeneity by incorporating random effects. All statistical tests were twotailed and $P<0.05$ were considered statistically significant.

\section{Results}

\subsection{Baseline characteristics of control subjects and participants with T2D}

Among all thirty-eight participants with T2D enrolled in this study, thirty-five participants completed the follow-up and three participants dropped out of this study because of poor glycemic control. No hypoglycemia occurred during the follow-up period. The baseline characteristics of the study participants were summarized in Table 1. Comparative analysis of baseline characteristics showed that there were no significant differences as regards age, sex, BMI, TC, LDL-C, TG and FINS levels between healthy controls and T2D participants (all $P>0.05$ ). Nevertheless, increased FBG, HbA1c and HOMA-IR as well as decreased HDL-C and HOMA- $\beta$ were observed in the T2D group compared with the healthy individuals (all $P<0.05)$. Serum adropin levels were significantly lower in the T2D group than those in the control group $(3.12 \pm 0.73$ vs. $5.90 \pm 1.22 \mathrm{ng} / \mathrm{ml}, P<0.01)$. 
Table 1

Baseline characteristics of the study participants

\section{Groups}

\begin{tabular}{|llll|} 
& Matched control $(\mathbf{n}=\mathbf{2 8})$ & Type 2 diabetes $(\mathbf{n}=\mathbf{3 5})$ & P value \\
\hline Age $($ year $)$ & $50.25 \pm 13.49$ & $50.31 \pm 13.43$ & 0.985 \\
Sex $(\mathrm{male} / \mathrm{female})$ & $16 / 12$ & $21 / 14$ & 0.819 \\
\hline $\mathrm{BMI}\left(\mathrm{kg} / \mathrm{m}^{2}\right)$ & $24.63 \pm 2.88$ & $25.67 \pm 3.10$ & 0.178 \\
\hline TC $(\mathrm{mmol} / \mathrm{L})$ & $4.84 \pm 0.99$ & $4.80 \pm 0.92$ & 0.880 \\
\hline LDL-C $(\mathrm{mmol} / \mathrm{L})$ & $2.92 \pm 1.01$ & $3.04 \pm 0.94$ & 0.660 \\
\hline HDL-C $(\mathrm{mmol} / \mathrm{L})$ & $1.32 \pm 0.37$ & $1.15 \pm 0.26$ & $0.042^{*}$ \\
\hline TG $(\mathrm{mmol} / \mathrm{L})$ & $1.44(0.89,2.14)$ & $1.80(1.24,2.27)$ & 0.148 \\
\hline FBG $(\mathrm{mmol} / \mathrm{L})$ & $5.00 \pm 0.61$ & $8.67 \pm 1.47$ & $<0.001^{*}$ \\
\hline FINS $(\mu \mathrm{IU} / \mathrm{mL})$ & $8.00(5.10,13.25)$ & $10.20(6.70,13.10)$ & 0.316 \\
\hline HbA1c $(\%)$ & $5.64 \pm 0.47$ & $8.04 \pm 0.74$ & $<0.001^{*}$ \\
\hline HOMA-IR & $1.76(1.10,2.99)$ & $4.19(2.21,5.56)$ & $<0.001^{*}$ \\
\hline HOMA- $\beta$ & $120.55(77.38,165.15)$ & $41.23(22.83,58.26)$ & $<0.001^{*}$ \\
\hline Adropin $(\mathrm{ng} / \mathrm{mL})$ & $5.90 \pm 1.22$ & $3.12 \pm 0.73$ & $<0.001^{*}$ \\
\hline
\end{tabular}

Abbreviations: BMI: body mass index; TC: total cholesterol; LDL-C: low-density lipoprotein cholesterol; HDL-C: high-density lipoprotein cholesterol; TG: triglyceride; FBG: fasting blood glucose; FINS: fasting insulin; HbA1c: glycosylated hemoglobin; HOMA-IR: homeostasis model assessment of insulin resistance; HOMA- $\beta$ : homeostasis model assessment of $\beta$-cell function.

Data shown as mean \pm standard deviation were compared between 2 groups using Student's t test for independent samples;

Data shown as median (interquartile range) were compared between 2 groups using Mann-Whitney Utest;

Data shown as $\mathrm{n}(\%)$ were compared between two groups using the chi-square test.

$\star P<0.05$

\subsection{Correlations between serum adropin levels and the baseline parameters}

In addition, the correlations between serum adropin levels and the baseline parameters were investigated in all participants. The serum adropin levels were significantly negatively correlated with TG, FBG, HbA1c 
and HOMA-IR (TG: $r=-0.271$; $P<0.05$; FBG: $r=-0.750, P<0.01$; HbA1c: $r=-0.770, P<0.01$; HOMA-IR: $r=$ $-0.441, P<0.01)$. Serum adropin levels were significantly positively correlated with HDL-C and HOMA- $\beta$ (HDL-C: $r=0.280, P<0.05$; HOMA- $\beta$ : $r=0.596, P<0.01$ ) (Table 2).

Table 2

Simple correlation analyses of serum adropin levels associated with other biochemical parameters in baseline

\begin{tabular}{|c|c|c|}
\hline \multicolumn{3}{|c|}{ Adropin } \\
\hline & $r$ & $P$ value \\
\hline Age & -0.055 & 0.669 \\
\hline BMI & -0.243 & 0.055 \\
\hline TC & 0.084 & 0.513 \\
\hline LDL-C & 0.079 & 0.539 \\
\hline HDL-C & 0.280 & $0.026^{\star}$ \\
\hline TG & -0.271 & $0.032 *$ \\
\hline FBG & -0.750 & $<0.001 *$ \\
\hline FINS & -0.152 & 0.236 \\
\hline $\mathrm{HbA1c}$ & -0.770 & $<0.001^{*}$ \\
\hline HOMA-IR & -0.441 & $<0.001^{*}$ \\
\hline HOMA- $\beta$ & 0.596 & $<0.001^{\star}$ \\
\hline \multicolumn{3}{|c|}{$\begin{array}{l}\text { Abbreviations: BMI: body mass index; TC: total cholesterol; LDL-C: low-density lipoprotein cholesterol, } \\
\text { HDL-C: high-density lipoprotein cholesterol; TG: triglyceride; FBG: fasting blood glucose; FINS: fasting } \\
\text { insulin; HbA1C: glycosylated hemoglobin; HOMA-IR: homeostasis model assessment of insulin } \\
\text { resistance; HOMA- } \beta \text { : homeostasis model assessment of } \beta \text {-cell function. }\end{array}$} \\
\hline$\star P<0.05$ & & \\
\hline
\end{tabular}

\subsection{Effect of sitagliptin monotherapy on serum adropin levels and other metabolic parameters in T2D group}

Changes in clinical parameters after sitagliptin treatment in T2D participants were summarized in Table 3. Sitagliptin treatment significantly decreased BMI, FBG, HbA1C and HOMA-IR compared with baseline (all $P<0.01)$. Moreover, HOMA- $\beta$ were significantly increased after sitagliptin treatment $(P<$ $0.01)$. But no obvious changes in lipid profiles (TC, LDL-C, HDL-C and TG) and FINS were observed $(P>$ 0.05). Importantly, the increases in adropin were observed after sitagliptin treatment compared to baseline $(4.97 \pm 1.01 \mathrm{vs} .3 .12 \pm 0.73 \mathrm{ng} / \mathrm{ml}, P<0.01)$. 
Table 3

Pre-treatment and post-treatment clinical characteristics of T2D participants treated with sitagliptin Group

\begin{tabular}{|c|c|c|c|c|}
\hline & $\begin{array}{l}\text { Pre-treatment } \\
(n=35)\end{array}$ & $\begin{array}{l}\text { Post-treatment } \\
(n=35)\end{array}$ & $\begin{array}{l}\text { Changes } \\
\text { after sitagliptin }\end{array}$ & $P$ value \\
\hline BMI $\left(\mathrm{kg} / \mathrm{m}^{2}\right)$ & $25.67 \pm 3.10$ & $25.12 \pm 3.14$ & $-0.55(-0.83,-0.26)$ & $<0.001$ * \\
\hline TC (mmol/L) & $4.80 \pm 0.92$ & $4.79 \pm 0.85$ & $-0.02(-0.23,0.20)$ & 0.881 \\
\hline LDL-C (mmol/L) & $3.04 \pm 0.94$ & $3.03 \pm 0.91$ & $-0.01(-0.23,0.21)$ & 0.938 \\
\hline $\mathrm{HDL}-\mathrm{C}(\mathrm{mmol} / \mathrm{L})$ & $1.15 \pm 0.26$ & $1.20 \pm 0.32$ & $0.05(-0.01,0.10)$ & 0.088 \\
\hline TG (mmol/L) & $1.80(1.24,2.27)$ & $1.39(1.05,2.34)$ & $-0.08(-0.42,0.27)$ & 0.101 \\
\hline FBG (mmol/L) & $8.67 \pm 1.47$ & $6.68 \pm 0.93$ & $-1.98(-2.47-1.49)$ & $<0.001^{*}$ \\
\hline FINS ( $\mu \mathrm{IU} / \mathrm{mL})$ & $10.20(6.70,13.10)$ & $7.80(5.40,12.40)$ & $-1.60(-3.48,0.28)$ & 0.177 \\
\hline HbA1c (\%) & $8.04 \pm 0.74$ & $6.59 \pm 0.53$ & $-1.45(-1.73,-1.17)$ & $<0.001^{*}$ \\
\hline HOMA-IR & $4.19(2.21,5.56)$ & $2.50(1.41,3.49)$ & $-1.56(-2.47,-0.65)$ & $<0.001^{*}$ \\
\hline HOMA- $\beta$ & $41.23(22.83,58.26)$ & $53.47(32.95,85.31)$ & $19.29(9.44,29.14)$ & $<0.001^{*}$ \\
\hline Adropin (ng/mL) & $3.12 \pm 0.73$ & $4.97 \pm 1.01$ & $1.85(1.47,2.23)$ & $<0.001^{*}$ \\
\hline \multicolumn{5}{|c|}{$\begin{array}{l}\text { Abbreviations: BMI: body mass index; TC: total cholesterol; LDL-C: low-density lipoprotein cholesterol; } \\
\text { HDL-C: high-density lipoprotein cholesterol; TG: triglyceride; FBG: fasting blood glucose; FINS: fasting } \\
\text { insulin; HbA1c: glycosylated hemoglobin; HOMA-IR: homeostasis model assessment of insulin } \\
\text { resistance; HOMA- } \beta \text { : homeostasis model assessment of } \beta \text {-cell function. }\end{array}$} \\
\hline \multicolumn{5}{|c|}{$\begin{array}{l}\text { Data shown as mean } \pm \text { standard deviation were compared between pre- and post-treatment using } \\
\text { paired Student's t test; }\end{array}$} \\
\hline \multicolumn{5}{|c|}{$\begin{array}{l}\text { Data shown as median (interquartile range) were compared between pre- and post-treatment using } \\
\text { paired Wilcoxon test; }\end{array}$} \\
\hline \multicolumn{5}{|l|}{$\star P<0.05$} \\
\hline
\end{tabular}

\subsection{Association between serum adropin levels and metabolic parameters after sitagliptin treatment}

Longitudinal analysis over the study period using linear mixed effects models was displayed in Fig. 1\&2. In multivariable models adjusted for sex, age, BMI and lipid profiles (TC, HDL-C, LDL-C and TG), higher serum adropin levels were associated with lower FBG $(\beta=-0.71, P<0.01$, Fig. $1 \mathrm{~A})$, lower HbA1c $(\beta=-0.44$, $P<0.01$, Fig. 1B), and higher HOMA- $\beta$ ( $\beta=9.02, P<0.01$, Fig. 1D). There is no statistical significance between HOMA-IR $(\beta=-0.35, P>0.05$, Fig. $1 \mathrm{C})$, FINS $(\beta=-0.06, P>0.05$, Fig. 1E). and serum adropin 
levels. Using other regression models adjusted for sex, age, BMI, FBG, HbA1c and FINS, lipid profiles were not significantly associated with serum adropin levels. (all $P>0.05$, Fig. 2A-D).

\section{Discussion}

The present study showed that serum adropin levels were lower in participants with newly diagnosed T2D than in the healthy controls. At baseline, serum adropin levels were negatively correlated with TG, FBG, HbA1c and HOMA-IR while positively correlated with HDL-C and HOMA- $\beta$. Importantly, sitagliptin treatment significantly increased serum adropin levels with the improvement of metabolic parameters. Furthermore, the associations of adropin with FBG, HbA1c and HOMA- $\beta$ still existed after sitagliptin treatment.

Adropin is a regulatory hormone produced mainly in the liver and brain, which is involved in glucolipid metabolism and insulin sensitivity ${ }^{2,3}$. Our study showed that the T2D participants had significantly lower adropin levels. Meanwhile, serum adropin levels were negatively TG, FBG, HbA1c and HOMA-IR while positively correlated with HDL-C and HOMA- $\beta$. These results were similar with other studies ${ }^{8,18}$. In animal studies, adropin overexpression or adropin treatment significantly ameliorated insulin resistance, enhanced glucose tolerance and improved glycolipid metabolism in diet-induced obese mice with insulin resistance ${ }^{3,4,19}$. Mechanistically, several studies have found that adropin can exert beneficial metabolic effects through different mechanisms. Firstly, it was reported that adropin has a direct role in the regulation of glucose metabolism. Adropin enhanced hepatic intracellular signaling actions that were involved in insulin-mediated glucose homeostasis. Adropin inhibited cAMP-PKA signaling pathway, leading to reduce the inositol triphosphate receptor (IP3R) phosphorylation and suppress the expression of cAMP-responsive element-binding protein (CREB) and CREB-regulated transcription co-activator 2 (CRTC2), which are two key factors in hepatic glucose metabolism ${ }^{3,4}{ }^{19}$. Next, adropin reduced the expression of genes involved in lipogenesis such as stearoyl-CoA desaturase-1 (SCD-1) and fatty acid synthase (Fas) in both liver and adipose tissue ${ }^{1}$. Therefore, these findings indicated that decreased adropin was closely related to the occurrence and development of T2D.

Consistent with previous studies, BMI, FBG, HbA1c and HOMA-IR significantly decreased and HOMA- $\beta$ significantly increased in T2D participants following sitagliptin treatment. However, sitagliptin treatment exhibited a greater reduction of BMI and $\mathrm{HbA} 1 \mathrm{c}$ in our study than that in other studies ${ }^{10,20}$. Several possible reasons that might explain this inconsistence. First, sitagliptin was employed as the initial therapy, meanwhile, lifestyle intervention was used as an add-on treatment. Second, Chinese adults with T2D have higher postprandial plasma glucose levels compared with Western participants ${ }^{21}$. Sitagliptin stimulates glucose-dependent insulin secretion and inhibits postprandial glucagon effectively, thus significantly lowering the postprandial glucose levels ${ }^{22}$. Besides, sitagliptin treatment obviously decreased HOMA-IR value and increased HOMA- $\beta$ value in our study. These results are supported by animal studies showing that DPP-4 inhibitors can improve insulin resistance and increase insulin sensitivity $23-26$. 
Sitagliptin treatment significantly increased serum adropin levels, which were parallel with the improving of $\mathrm{FBG}, \mathrm{HbA} 1 \mathrm{C}$ and $\mathrm{HOMA}-\beta$. In animal studies, adropin overexpression significantly reduced insulin resistance and improved glucose tolerance in obese high-fat-fed mice ${ }^{1}$. Moreover, adropin treatment reduced blood glucose levels, HbA1c and HOMA-IR and increased HOMA- $\beta$ in a rat model of T2D ${ }^{19}$. However, the exact mechanism by which sitagliptin increases serum adropin levels remains unclear. But we found that sitagliptin and adropin had similar effects on the regulation of glucose metabolism in previous studies. DPP-4 inhibitor decreased gluconeogenic gene expression through the inhibition of CREB phosphorylation and CRTC2 expression in mice with diabetes ${ }^{4,26}$. Based on these similar findings, we believed that the upregulation of adropin might be a potential novel mechanism for beneficial effects of sitagliptin in T2D.

But several limitations in the present study should be noted. First, our study was not a randomized controlled trial, the causality between adropin and sitagliptin cannot infer. Randomized-controlled trials are needed to further confirm the beneficial effects we reported. Second, our findings are limited by a relatively small sample size, so we need to expand the sample size to support. Moreover, more animal and cell experiments are needed to reveal the underlying molecular mechanism of sitagliptin on adropin.

\section{Conclusion}

In conclusion, our study demonstrated that serum adropin levels were lower in newly diagnosed T2D participants. Sitagliptin treatment significantly increased serum adropin in the T2D group. And the changes in serum adropin level were parallel with the amelioration of glucose metabolism.

\section{Abbreviations}

Type 2 diabetes: T2D; FBG: fasting blood glucose; HbA1c: glycosylated hemoglobin; HOMA- $\beta$ : homeostasis model assessment of $\beta$-cell function; Echo: Energy Homeostasis Associated; NAFLD: nonalcoholic fatty liver disease; PCOS: polycystic ovary syndrome; DPP-4: dipeptidyl peptidase-4; OGTT: oral glucose tolerance test; BMI: body mass index; ADA: American Diabetes Association; FINS: fasting insulin; TC: total cholesterol; LDL-C: low-density lipoprotein cholesterol; HDL-C: high-density lipoprotein cholesterol; TG: triglyceride; ELISA: enzyme-linked immunosorbent assay; HOMA-IR: homeostasis model assessment of insulin resistance; SD: standard deviation; IP3R: inositol triphosphate receptor; CREB: cAMP-responsive element-binding protein; CRTC-2: CREB-regulated transcription co-activator 2; SCD-1: stearoyl-CoA desaturase-1: Fas.

\section{Declarations}

\section{Ethics approval and consent to participate}

The study was approved by the Ethics Committee of Beijing Chao-yang Hospital affiliated with Capital Medical University (2020-\#-182) and was registered at Clinicaltrials.gov (NCT number: NCT04495881). 
The protocol was implemented in accordance with provisions of the Declaration of Helsinki. All enrolled participants provided written informed consent in this study.

\section{Consent for publication}

Not applicable

\section{Availability of data and materials}

The datasets used and/or analysed during the current study are available from the corresponding author on reasonable request.

\section{Competing interests}

The authors declare that they have no competing interests.

\section{Funding}

This study was financially supported by the Chinese National Natural Science Foundation [No. 81770792] and Key Projects of Science and Technology Planning of Beijing Municipal Education Commission [KZ201810025038] to G.W. and the Beijing Talents foundation [2018-12] to J.L.

\section{Author's Contributions}

J.L. and G.W. conceived and designed the study, Q.W. conducted the experiments, performed the analyses and wrote the manuscript. Y.A., L.Z. and Y.Z. helped collect and analyze the data. All authors read and approved the final manuscript.

\section{Acknowledgements}

Not applicable

\section{References}

1. Kumar KG, Trevaskis JL, Lam DD, et al. Identification of adropin as a secreted factor linking dietary macronutrient intake with energy homeostasis and lipid metabolism. Cell Metab. Dec 2008;8(6):46881. doi:10.1016/j.cmet.2008.10.011

2. Ganesh Kumar K, Zhang J, Gao S, et al. Adropin deficiency is associated with increased adiposity and insulin resistance. Obesity (Silver Spring). Jul 2012;20(7):1394-402. doi:10.1038/oby.2012.31

3. Gao S, McMillan RP, Zhu Q, Lopaschuk GD, Hulver MW, Butler AA. Therapeutic effects of adropin on glucose tolerance and substrate utilization in diet-induced obese mice with insulin resistance. $\mathrm{Mol}$ Metab. Apr 2015;4(4):310-24. doi:10.1016/j.molmet.2015.01.005

4. Gao S, Ghoshal S, Zhang L, et al. The peptide hormone adropin regulates signal transduction pathways controlling hepatic glucose metabolism in a mouse model of diet-induced obesity. J Biol 
Chem. Sep 6 2019;294(36):13366-13377. doi:10.1074/jbc.RA119.008967

5. Sayin O, Tokgoz Y, Arslan N. Investigation of adropin and leptin levels in pediatric obesity-related nonalcoholic fatty liver disease. J Pediatr Endocrinol Metab. May 2014;27(5-6):479-84. doi:10.1515/jpem-2013-0296

6. Kume T, Calan M, Yilmaz O, et al. A possible connection between tumor necrosis factor alpha and adropin levels in polycystic ovary syndrome. J Endocrinol Invest. Jul 2016;39(7):747-54. doi:10.1007/s40618-016-0453-5

7. Yosaee S, Khodadost M, Esteghamati A, et al. Metabolic Syndrome Patients Have Lower Levels of Adropin When Compared With Healthy Overweight/Obese and Lean Subjects. Am J Mens Health. Mar 2017;11(2):426-434. doi:10.1177/1557988316664074

8. Zang H, Jiang F, Cheng X, Xu H, Hu X. Serum adropin levels are decreased in Chinese type 2 diabetic patients and negatively correlated with body mass index. Endocr J. Jul 28 2018;65(7):685-691. doi:10.1507/endocrj.EJ18-0060

9. Zheng Y, Ley SH, Hu FB. Global aetiology and epidemiology of type 2 diabetes mellitus and its complications. Nat Rev Endocrinol. Feb 2018;14(2):88-98. doi:10.1038/nrendo.2017.151

10. Ahren B. Use of DPP-4 inhibitors in type 2 diabetes: focus on sitagliptin. Diabetes Metab Syndr Obes. Mar 29 2010;3:31-41. doi:10.2147/dmsott.s7327

11. Zillessen P, Celner J, Kretschmann A, Pfeifer A, Racke K, Mayer P. Metabolic role of dipeptidyl peptidase 4 (DPP4) in primary human (pre)adipocytes. Sci Rep. Mar 17 2016;6:23074. doi:10.1038/srep23074

12. Baumeier C, Saussenthaler S, Kammel A, et al. Hepatic DPP4 DNA Methylation Associates With Fatty Liver. Diabetes. Jan 2017;66(1):25-35. doi:10.2337/db15-1716

13. Mi DH, Fang HJ, Zheng GH, et al. DPP-4 inhibitors promote proliferation and migration of rat brain microvascular endothelial cells under hypoxic/high-glucose conditions, potentially through the SIRT1/HIF-1/VEGF pathway. CNS Neurosci Ther. Mar 2019;25(3):323-332. doi:10.1111/cns.13042

14. Wang Q, Ma L, Zhang Y, et al. Effect of Sitagliptin on Serum Irisin Levels in Patients with Newly Diagnosed Type 2 Diabetes Mellitus. Diabetes Ther. Apr 2021;12(4):1029-1039. doi:10.1007/s13300021-01023-z

15. American Diabetes A. Standards of medical care in diabetes--2014. Diabetes Care. Jan 2014;37 Suppl 1:S14-80. doi:10.2337/dc14-S014

16. Katsuki A, Sumida Y, Gabazza EC, et al. Homeostasis model assessment is a reliable indicator of insulin resistance during follow-up of patients with type 2 diabetes. Diabetes Care. Feb 2001;24(2):362-5. doi:10.2337/diacare.24.2.362

17. Bermudez V, Cano R, Cano C, et al. Homeostasis model assessment (HOMA) as surrogate insulinization criteria in patients with type 2 diabetes. Am J Ther. Jul-Aug 2008;15(4):409-16. doi:10.1097/MJT.0b013e318160b909

18. Wu L, Fang J, Chen L, et al. Low serum adropin is associated with coronary atherosclerosis in type 2 diabetic and non-diabetic patients. Clin Chem Lab Med. May 2014;52(5):751-8. doi:10.1515/cclm- 
2013-0844

19. Akcilar R, Kocak FE, Simsek H, et al. Antidiabetic and hypolipidemic effects of adropinin streoptozotocin-induced type 2 diabetic rats. Bratis/ Lek Listy. 2016;117(2):100-5. doi:10.4149/bll_2016_020

20. Nonaka K, Kakikawa T, Sato A, et al. Efficacy and safety of sitagliptin monotherapy in Japanese patients with type 2 diabetes. Diabetes Res Clin Pract. Feb 2008;79(2):291-8.

doi:10.1016/j.diabres.2007.08.021

21. Herman $\mathrm{WH}$, Dungan $\mathrm{KM}$, Wolffenbuttel $\mathrm{BH}$, et al. Racial and ethnic differences in mean plasma glucose, hemoglobin A1c, and 1,5-anhydroglucitol in over 2000 patients with type 2 diabetes. $J$ Clin Endocrinol Metab. May 2009;94(5):1689-94. doi:10.1210/jc.2008-1940

22. Deacon CF. Physiology and Pharmacology of DPP-4 in Glucose Homeostasis and the Treatment of Type 2 Diabetes. Front Endocrinol (Lausanne). 2019;10:80. doi:10.3389/fendo.2019.00080

23. Kulkarni RN, Bruning JC, Winnay JN, Postic C, Magnuson MA, Kahn CR. Tissue-specific knockout of the insulin receptor in pancreatic beta cells creates an insulin secretory defect similar to that in type 2 diabetes. Cell. Feb 5 1999;96(3):329-39. doi:10.1016/s0092-8674(00)80546-2

24. Shirakawa J, Amo K, Ohminami H, et al. Protective effects of dipeptidyl peptidase-4 (DPP-4) inhibitor against increased beta cell apoptosis induced by dietary sucrose and linoleic acid in mice with diabetes. J Biol Chem. Jul 22 2011;286(29):25467-76. doi:10.1074/jbc.M110.217216

25. Shirakawa J, Fujii $\mathrm{H}$, Ohnuma K, et al. Diet-induced adipose tissue inflammation and liver steatosis are prevented by DPP-4 inhibition in diabetic mice. Diabetes. Apr 2011;60(4):1246-57. doi:10.2337/db10-1338

26. Kim TH, Kim MK, Cheong YH, et al. Hepatic role in an early glucose-lowering effect by a novel dipeptidyl peptidase 4 inhibitor, evogliptin, in a rodent model of type 2 diabetes. Eur $J$ Pharmacol. Jan 15 2016;771:65-76. doi:10.1016/j.ejphar.2015.11.029

\section{Figures}



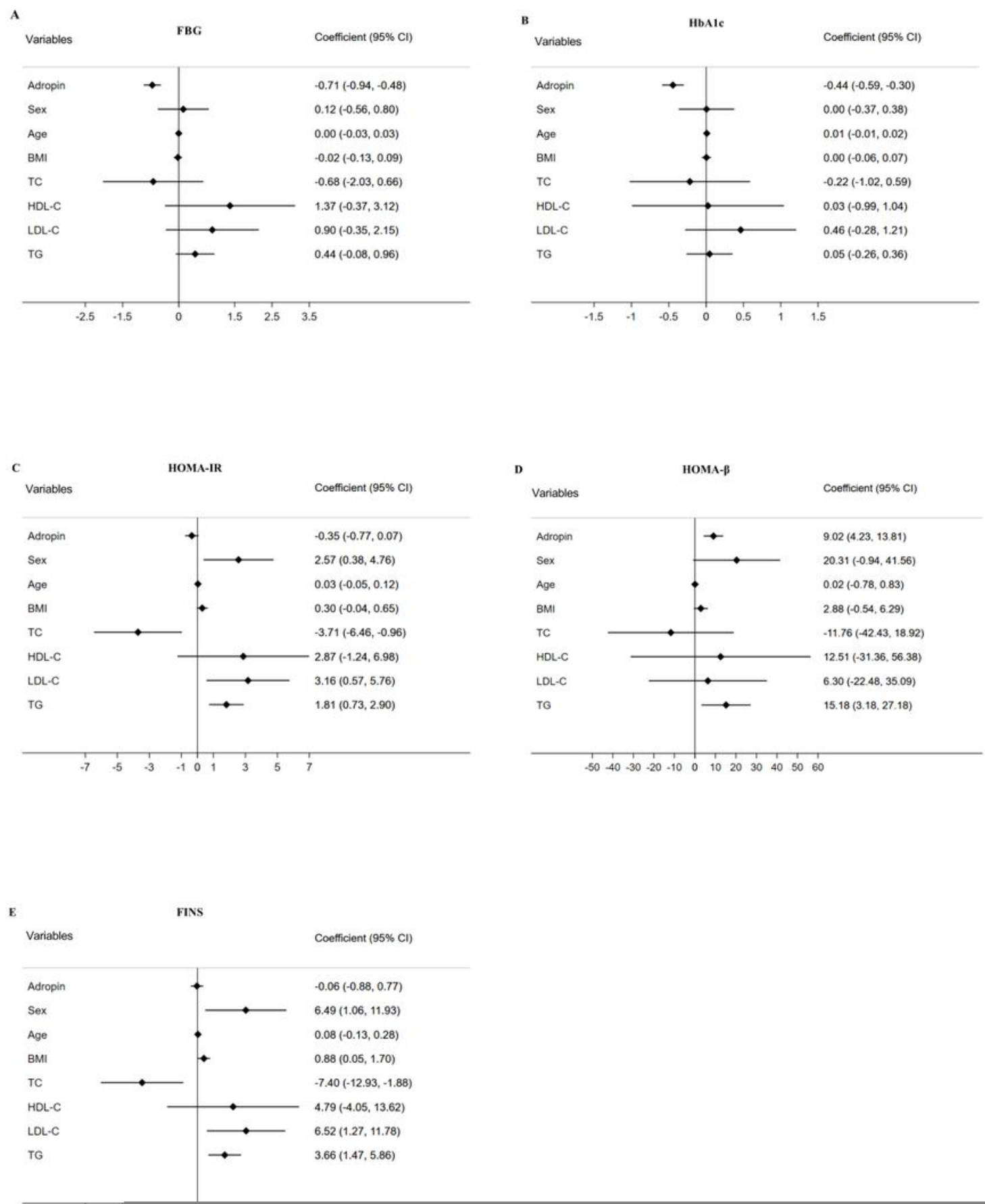

\section{Figure 1}

Forest plots demonstrating associations between adropin and FBG (A), HbA1c (B), HOMA-IR (C), HOMA- $\beta$ (D) and FINS (E) in mixed-effect linear models adjusted for potential confounders. Abbreviations: FBG: fasting blood glucose; HbA1c: glycosylated hemoglobin; HOMA-IR: homeostasis model assessment of insulin resistance; HOMA- $\beta$ : homeostasis model assessment of $\beta$-cell function; FINS: fasting insulin; BMI: 
body mass index; TC: total cholesterol; HDL-C: high-density lipoprotein cholesterol; LDL-C: low-density lipoprotein cholesterol; TG: triglyceride.
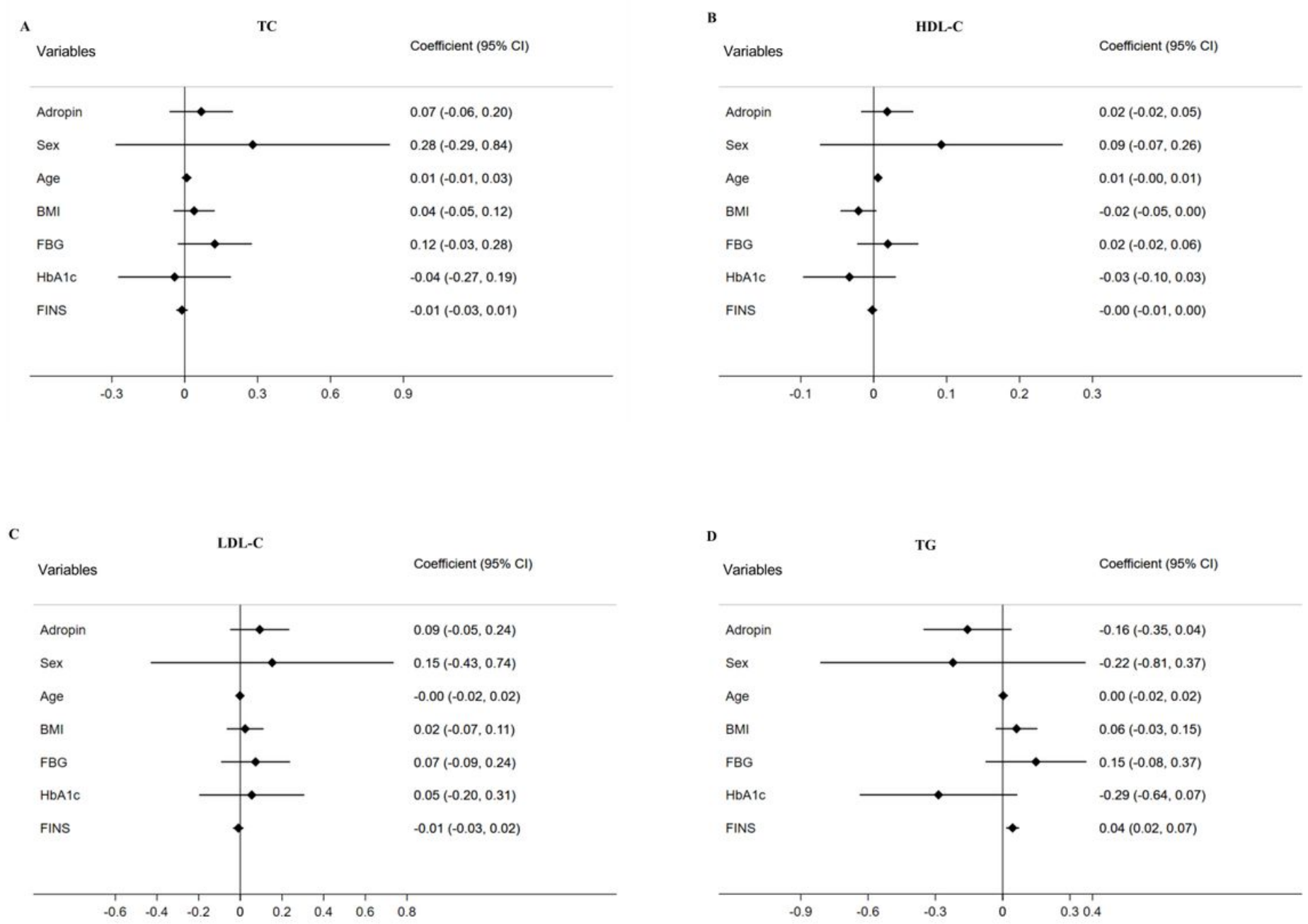

Figure 2

Forest plots demonstrating associations between adropin and TC (A), HDL-C (B), LDL-C (C) and TG (D) in mixed-effect linear models adjusted for potential confounders. Abbreviations: TC: total cholesterol; HDLC: high-density lipoprotein cholesterol; LDL-C: low-density lipoprotein cholesterol; TG: triglyceride; BMI: body mass index; FBG: fasting blood glucose; HbA1c: glycosylated hemoglobin; FINS: fasting insulin. 